\title{
DIRECT IMAGES OF HERMITIAN HOLOMORPHIC BUNDLES
}

\author{
H. GILLET AND C. SOULÉ
}

Introduction. We introduce higher analogues of analytic torsion, which are form valued. Using this construction we obtain, in the case of the projection map for a product, a Grothendieck-Riemann-Roch theorem for hermitian holomorphic vector bundles which is an equality between differential forms. This is related to work of Quillen [6] and of Bismut and Freed [1].

\section{A Grothendieck group.}

I.1. Let $X$ be a complex manifold. For any $p \in \mathbf{N}$ let $A^{p, p}(X)$ be the space of real $(p, p)$ forms over $X$. Let $A(X)=\bigoplus_{p>0} A^{p, p}(X)$, and $\tilde{A}(X)=$ $A(X) /(\operatorname{Im}(\partial)+\operatorname{Im}(\bar{\partial}))$, where $d=\partial+\bar{\partial}$ is the standard decomposition of the exterior derivative on $X$.

I.2. An hermitian holomorphic bundle (or h.h. bundle) on $X$ is a pair $\bar{E}=(E, h)$, consisting of a finite-dimensional complex holomorphic vector bundle $E$ over $X$ and a smooth hermitian scalar product $h$ on $E$. Given $\bar{E}$, let $\nabla$ be the unique connection on $E$ which is both compatible with its complex structure and unitary for $h$, as in [2]. The closed form $\operatorname{ch}(\bar{E})=$ $\operatorname{Tr}\left(\exp \left((i / 2 \pi) \nabla^{2}\right)\right.$ in $A(X)$ represents the Chern character of $E$.

I.3. Let $\hat{K}_{0}(X)$ be the abelian group generated by pairs $(\bar{E}, \eta)$ where $E$ is an h.h. bundle over $X$ and $\eta \in \tilde{A}(X)$, with the following relations. Let

$$
\bar{\varepsilon}: 0 \rightarrow \bar{S} \rightarrow \bar{E} \rightarrow \bar{Q} \rightarrow 0
$$

be any exact sequence of holomorphic bundles over $X$, endowed with arbitrary metrics, and $\eta^{\prime}, \eta^{\prime \prime} \in \tilde{A}(X)$. We impose the relation $\left(\bar{S} ; \eta^{\prime}\right)+\left(\bar{Q} ; \eta^{\prime \prime}\right)=$ $\left(\bar{E} ; \eta^{\prime}+\eta^{\prime \prime}-\widetilde{\operatorname{ch}}(\overline{\mathcal{E}})\right)$, where $\widetilde{\mathrm{ch}}(\overline{\mathcal{E}}) \in \tilde{A}(X)$ is the solution to the equation

$$
(1 / \pi i) \partial \bar{\partial} \tilde{\operatorname{ch}}(\overline{\mathcal{E}})=\operatorname{ch}(\bar{S})+\operatorname{ch}(\bar{Q})-\operatorname{ch}(\bar{E})
$$

introduced by Bott and Chern in [2].

I.4. The following construction of $\tilde{\mathrm{ch}}$ is used in the proofs of the results below. Let $O(1)$ be the tautological line bundle on the complex projective line $\mathbf{P}^{1}$, and let $z$ be the parameter on the affine line $\mathbf{A}^{1} \subset \mathbf{P}^{1}$. If $\sigma: O \rightarrow O(1)$ is the section vanishing at infinity, let $s=\operatorname{Id} \otimes \sigma$ be the induced map $S \rightarrow S(1)$ on $X \times \mathbf{P}^{1}$. If $i: S \rightarrow E$ is the inclusion in $\mathcal{E}$ above, let $F=(S(1) \oplus E) / S$ be the vector bundle which is the cokernel of $s \oplus i$. If $i_{p}: X \times\{p\} \rightarrow X \times \mathbf{P}^{1}$ for $p=0, \infty$ are the natural inclusions, then $i_{0}^{*} F \simeq E$ while $i_{\infty}^{*} F \simeq S \oplus Q$. We may choose a metric on $F$ so that these maps are isometries. Then, in $\tilde{A}(X)$ :

$$
\widetilde{\operatorname{ch}}(\overline{\mathcal{E}})=\int_{z} \operatorname{ch}(\bar{F}) \log |z| .
$$

Received by the editors May 8, 1986.

1980 Mathematics Subject Classification (1985 Revision). Primary 58G10.

Partially supported by NSF Grant DMS 850248. 
I.5. Define a homomorphism ch: $\widehat{K_{0}}(X) \rightarrow A(X)$ by setting

$$
\operatorname{ch}(\bar{E} ; \eta)=\operatorname{ch}(\bar{E})-(1 / \pi i) \partial \bar{\partial} \eta \text {. }
$$

I.6. When $X$ is algebraic over a ring $R$ contained in $\mathbf{C}$ one can require, when defining $\widehat{K_{0}}(X)$, that the bundles be algebraic over $R$ (cf. $[3]$ ). The results below remain true in that case.

\section{Higher analytic torsion.}

II.1. Let $X$ be a complex manifold, $Y$ a compact Kähler manifold and $f: X \times Y \rightarrow X$ the projection onto the first factor.

Let $\bar{E}$ be an h.h. bundle over $X \times Y$. For any point $x$ in $X$, we get an h.h. bundle $\bar{E}_{x}$ on $Y$ by restricting $\bar{E}$ to the fibre $f^{-1}(x) \simeq Y$. In this section we assume that

(A) For all $x$ in $X$ and all $k>0$, the cohomology group $H^{k}\left(Y, E_{x}\right)$ vanishes. Under this assumption, we shall define a form $T(f, \bar{E})$ in $\tilde{A}(X)$, called the higher analytic torsion of $E$ (relative to $f$ ).

II.2. From (A) we know that there is a vector bundle $f_{*}(E)$ on $X$ the fibre of which at any $x \in X$ is equal to $H^{0}\left(Y, E_{x}\right)$. For any $q \in \mathbf{N}$ let $T^{*}(Y)^{0, q}$ be $(0, q)$ part of the $q$ th exterior power of the complexified cotangent bundle to $Y$. Let $D^{q}$ be the smooth infinite-dimensional bundle over $X$, with fiber at $x \in X$ equal to the space of smooth sections over $Y$ of the bundle $E_{x} \otimes T^{*}(Y)^{0, q}$. The Dolbeault resolution of $E_{x}$ over $Y$, as $x \in X$ varies, gives rise to an acyclic complex of bundles on $X$ :

$$
0 \rightarrow f_{*}(E) \rightarrow D^{0} \rightarrow D^{1} \rightarrow D^{2} \rightarrow \cdots .
$$

II.3. The choice of metrics on $Y$ and $E$ determines a metric on $E_{x} \otimes$ $T^{*}(Y)^{0, q}$ and an $L^{2}$ scalar product on $D^{q}, q \geq 0$. Let $f_{*}(h)$ be the hermitian metric on $f_{*}(E)$ induced from $D^{0}$, and $\nabla$ the associated connection (I.2). We also define a connection $\nabla$ on $D^{q}, q \geq 0$, as follows. Giving a section $s$ of $D^{q}$ over $X$ is equivalent to giving a section of the bundle $E \otimes \pi^{*}\left(T^{*}(Y)^{0, q}\right)$ over $X \times Y$, where $\pi: X \times Y \rightarrow Y$ is the second projection. The latter bundle has a metric and therefore a connection $\tilde{\nabla}$. If $v$ is a tangent vector to $X$ at the point $x=f(x, y)$, let $\tilde{v}$ be the horizontal tangent vector to $X \times Y$ at $(x, y)$ such that $f(\tilde{v})=v$. We define $\nabla_{v}(s)(x, y)$ to be $\tilde{\nabla}_{\tilde{v}}(s)(x, y)$.

II.4. We write $H^{+}$for the pull-back from $X$ to $X \times \mathbf{C}^{*}$ of $\bigoplus_{i \geq 0} D^{2 i}$, and $H^{-}$for the pull back of $\bigoplus_{i \geq 0} D^{2 i+1} \oplus f_{*}(E)$. These bundles admit the connection

$$
\nabla_{z}=\nabla+\frac{\partial}{\partial z} d z+\frac{\partial}{\partial \bar{z}} d \bar{z}
$$

where $z$ is the coordinate in $\mathbf{C}^{*}$. Let $\bar{\partial}_{Y}^{*}$ (resp. $j^{*}$ ) be the adjoint of $\bar{\partial}_{Y}$ (resp. $j)$, and $L_{z}: H^{+} \oplus H^{-} \rightarrow H^{+} \oplus H^{-}$be the odd endomorphism $i\left(z \bar{\partial}_{Y} \oplus \bar{z} \bar{\partial}_{Y}^{*} \oplus\right.$ $\left.z j \oplus \bar{z} j^{*}\right)$. Following Quillen [5], we give the super vector bundle $H^{+} \oplus H^{-}$ the superconnection $\nabla_{z}+L_{z}$. The operator $\exp \left(\nabla_{z}+L_{z}\right)^{2}$ is trace class on $\mathrm{H}^{+} \oplus \mathrm{H}^{-}$and its supertrace

$$
\omega(z)=\operatorname{tr}_{s} \exp \left(\nabla_{z}+L_{z}\right)^{2}
$$


is a form on $X \times \mathbf{C}^{*}$ (compare [5 and 1]). For any real number $r>0$ one can show that the integral

$$
I(r)=\int_{|z|^{2}>r} \omega(z) \log |z|
$$

converges, belongs to $A(X)$, and admits an asymptotic development (as $r$ goes to zero) with finitely many divergences of type $r^{-j-1}$ and $r^{-j} \log (r), j \in \mathbf{N}$. Let $I(0) \in A(X)$ be the finite part of $I(r)$ as $r$ goes to zero. By definition, $T(f, \bar{E}) \in \tilde{A}(X)$ is the class of the form obtained from $I(0)$ by multiplying its $(p, p)$ component by $(i / 2 \pi)^{p+1}$, for all $p \geq 0$.

\section{A Riemann-Roch Theorem.}

III.1. Assume $X, Y$ and $\bar{E}$ are as in $\S I I$, and also that $Y$ is projective. Let $f_{!}(\bar{E}) \in \widehat{K_{0}}(X)$ be the class of $\left(f_{*}(E), f_{*}(h) ; T(f, \bar{E})\right)$. Let $T d(\bar{Y}) \in A(Y)$ denote the closed form, representing the Todd class of $Y$, determined by the choice of metric on $Y$, as in I.2. For any $\eta \in \tilde{A}(X \times Y)$ let $f_{!}(0 ; \eta)=$ $\left(0 ; f_{*}(\eta T d(\bar{Y}))\right.$ in $\widehat{K_{0}}(X)$, where $f_{*}$ denotes integration along $Y$.

III.2. THEOREM 1. (i) The map $f_{\text {! }}$ extends uniquely to a group homomorphism

$$
f_{!}: \widehat{K_{0}}(X \times Y) \rightarrow \widehat{K_{0}}(X) .
$$

(ii) For any $\alpha$ in $\widehat{K_{0}}(X \times Y)$, we have an equality in $A(X)$ :

$$
\operatorname{ch}\left(f_{!}(\alpha)\right)=f_{*}(\operatorname{ch}(\alpha) T d(\bar{Y})) .
$$

\section{The metric on the determinant bundle.}

IV.1. Let $\widehat{\mathrm{Pic}}(X)$ be the group of hermitian holomorphic line bundles over $X$, modulo biholomorphic isometries. There is a morphism

$$
\widehat{\operatorname{det}}: \widehat{K_{0}}(X) \rightarrow \widehat{\operatorname{Pic}}(X)
$$

sending $(E, h: \eta)$ to the maximal exterior power $L=\bigwedge^{\max }(E)$, given the metric $\bigwedge^{\max }(h) \exp \left(-2 \eta^{0}\right)$, where $\eta^{0} \in C^{\infty}(X, \mathbf{R})$ is the component of $\eta$ of degree zero.

IV.2. Let $X$ and $Y$ be as in II.1 and let $\bar{E}$ be an h.h. bundle over $X \times Y$. On the line bundle $\lambda(E)=\operatorname{det} R f_{*}(E)$ over $X$ (cf. [4]) we define a metric $h$ as follows. For any point $x \in X$, let $\Delta^{q}$ be the Laplace operator $\bar{\partial}_{Y} \bar{\partial}_{Y}^{*}+\bar{\partial}_{Y}^{*} \bar{\partial}_{Y}$ on $D_{x}^{q}, q \geq 0, H_{x}^{q}$ its kernel, and $K_{x}^{q}$ the orthogonal complement to $H_{x}^{q}$ in $D_{x}^{q}$. When $s \in \mathbf{C}$ has large enough real part, consider the zeta function $\varsigma^{q}(s)=\operatorname{Trace}\left(\left(\Delta^{q}\right)^{-s}\right.$ on $\left.K_{x}^{q}\right)$. Now $\zeta^{q}(s)$ admits a meromorphic continuation to the whole complex plane, which is regular at the origin. Let

$$
\tau(x)=\sum_{q \geq 0}(-1)^{q}\left[q\left(\zeta^{q}\right)^{\prime}(0)-q \gamma \zeta^{q}(0)+(1-q) \gamma \operatorname{dim}_{\mathbf{C}} H_{x}^{q}\right]
$$

where $\gamma$ is the Euler constant. The $L^{2}$ metric on $H_{x}^{q}$ and the canonical isomorphism between $\lambda(E)_{x}$ and $\bigotimes_{q \geq 0}\left(\bigwedge^{\max }\left(H_{x}^{q}\right)\right)^{(-1)^{q}},[4]$, define a metric $h_{L^{2}}$ on $\lambda(E)_{x}$. Let $h_{Q}=h_{L^{2}} \exp (-\tau(x))$. One can show that the scalar product $h_{Q}$ on $\lambda(E)_{x}$ varies smoothly with $x$; see [6 and $\mathbf{1}$ ]. 
IV.3. THEOREM 2. If $\bar{E}$ satisfies II.1(A), the class of $\left(\lambda(E), h_{Q}\right)$ in $\widehat{\operatorname{Pic}}(X)$ is equal to $\widehat{\operatorname{det}}\left(f_{!}(\bar{E})\right)$.

IV.4. Theorems 1 and 2 imply that the first Chern form of $\left(\lambda(E), h_{Q}\right)$ is the component of degree two in $f_{*}(\operatorname{ch}(\bar{E}) T d(\bar{Y}))$. The proof of Theorem 1 uses the constructions of I and II, and the local index theorem of Bismut [7].

\section{REFERENCES}

1. J.-M. Bismut and D. Freed, The analysis of elliptic families, preprint (1985), Orsay; Fibré déterminant et invariant êta, C. R. Acad. Sci. Paris Sér. I 301 (1985), 707-710.

2. R. Bott and S. S. Chern, Hermitian vector bundles and the equidistribution of the zeroes of their holomorphic cross sections, Acta Math. 114 (1968), 71-112.

3. H. Gillet and C. Soulé, Classes caractéristiques en théorie d'Arakelov, C. R. Acad. Sci. Paris Sér. I 301 (1985), 439-442.

4. F. Knudsen and D. Mumford, The projectivity of the moduli space of stable curves. I, Math. Scand. 39 (1976), 19-55.

5. D. Quillen, Super connections and the Chern character, Topology 24 (1985), 89-95.

6. __ Determinants of Cauchy-Riemann operators over a Riemann surface, Functional Analysis 19 (1985), 3134.

7. J.-M. Bismut, The Atiyah-Singer index theorem for families of Dirac operators: two heat equation proofs, Invent. Math. 83 (1986), 91-152.

Department of MATHEMATICS, UNiversity of Illinois at Chicago, CHICAGO, ILLINOIS 60638

U.E.R. DE Mathématiques, UniVersité PaRis VII, TOUR NO. 45-55, 2, PLACE JUSSIEU, 75251 PARIS CEDEX 05, FRANCE 